\title{
la catedral de COVENTRY
}

SIR BASIL SPENCE, arquitecto

sinopsis

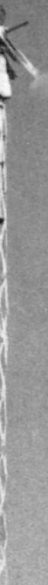
La nueva catedral de Coventry es un edificio de linea moderna y ponsentados y para 2.000 cuando se llena totalmente la nave central; mide aproximadamente $82,36 \mathrm{~m}$ de longitud y $24,40 \mathrm{~m}$ de anchura. La entrada principal se realiza por el porche a través de los huecos existentes en la gran mampara de cristal, grabada por John Hutton, que permite la clara visión del interior del recinto. A la derecha, entrando, se encuentra el baptisterio-iluminado por una vidriera ricamente policromada-; y a la izquierda, la capilla de la Unidad-de planta en forma de estrella- La nave central fue proyectada con la idea de conseguir la concentración de la atención de los fieles hacia el altar, lo que se ha direccional dacio a la iluminación. Detrás del presbiterio se encuentran las capillas, conocidas con el nombre de: *La Señoras, de *Cristo en Gethsemaniw $y$ de wLa Industria*. La eripta aloja: la sala capitular, la sacristia, despachos, dependencias auxiliares, etc. Entre las fachadas exteriores, en situación prominente, aparecen las esculturas de San Miguel $y$ el demonio, obras de Sir Jacob Epstein. Corona el edificio la arguja», de diseño abstracto, que se eleva $24 \mathrm{~m}$ sobre la parte superior de la cubierta, como una fiel expresión del espiritu de resurgimiento religioso en Inglaterra. 


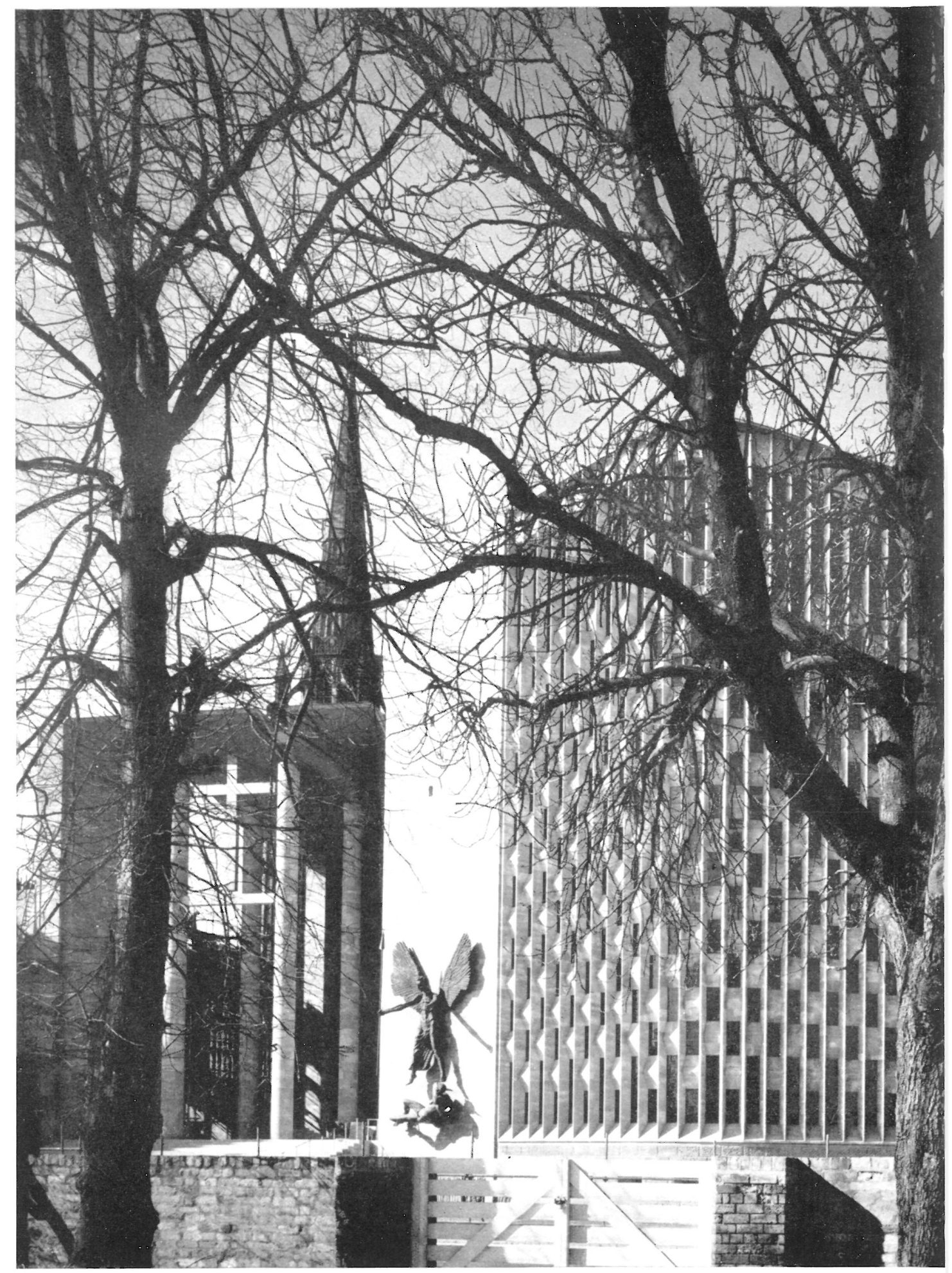

148.87

El nuevo edificio para la catedral de Coventry-construido según el proyecto elegido en un concurso celebrado años antes-se alza sobre una planta de eje perpendicular a las ruinas de la antigua catedral de San Miguel, que fue edificada en el siglo XV y destruida durante la última guerra.

Históricamente correcto y contemporáneamente apropiado, tiene capacidad para 1.350 fieles sentados, y para 2.000 cuando se llena totalmente la nave central; mide, aproximadamente, $82,36 \mathrm{~m}$ de longitud y $24,40 \mathrm{~m}$ de anchura. 

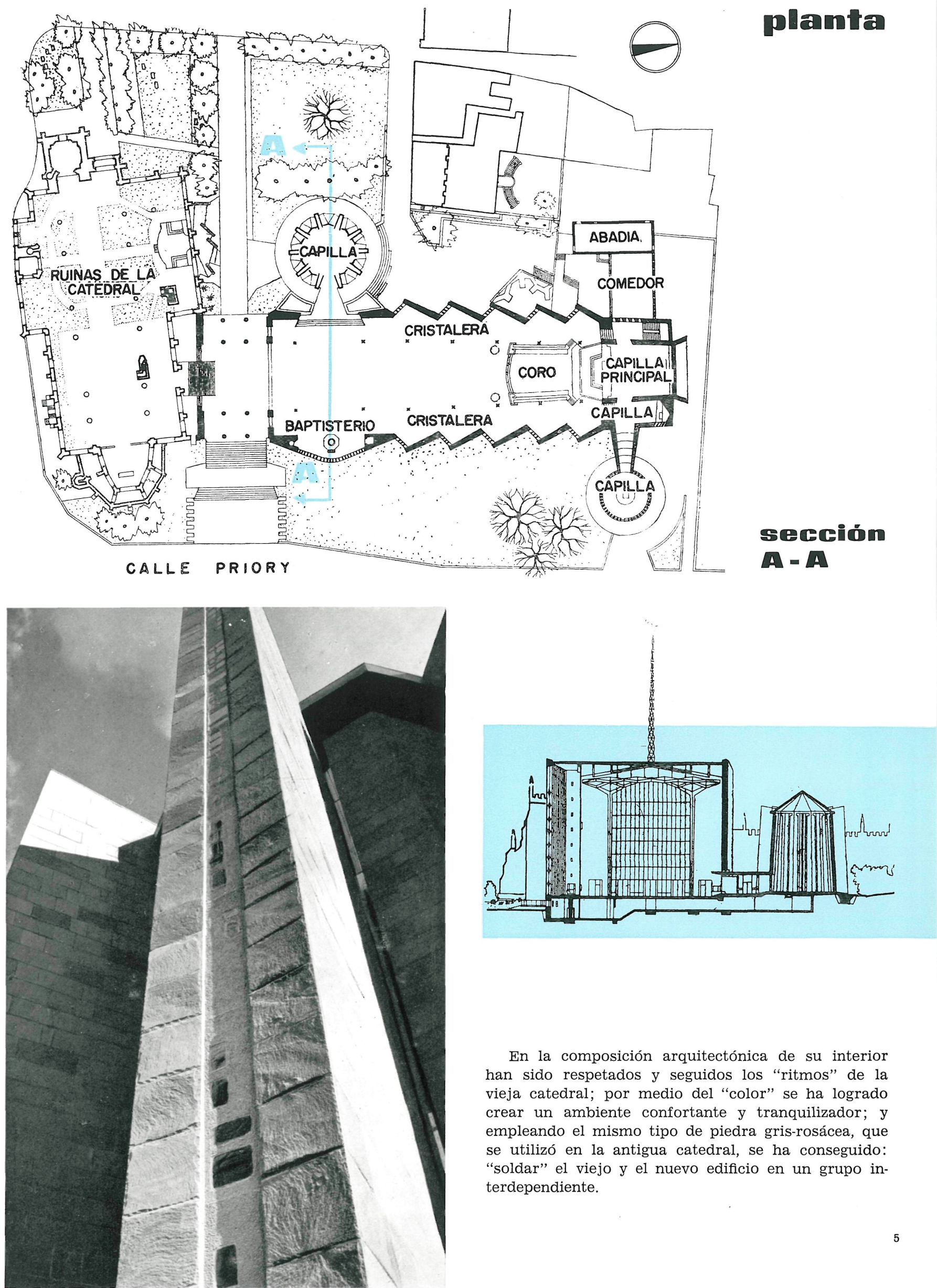

En la composición arquitectónica de su interior han sido respetados y seguidos los "ritmos" de la vieja catedral; por medio del "color" se ha logrado crear un ambiente confortante y tranquilizador; y empleando el mismo tipo de piedra gris-rosácea, que se utilizó en la antigua catedral, se ha conseguido: "soldar" el viejo y el nuevo edificio en un grupo interdependiente. 


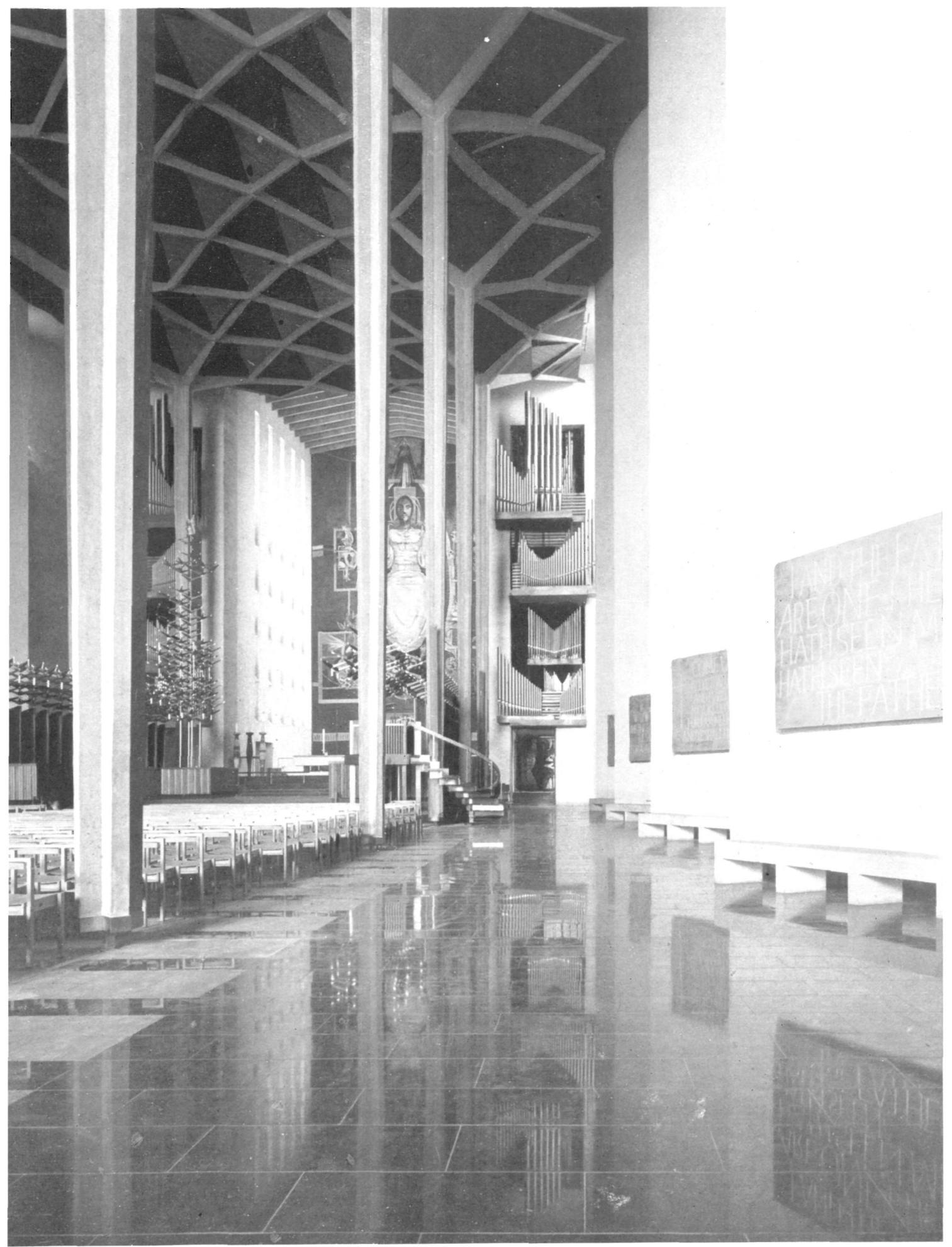

La entrada principal se realiza por el porche, a través de las aberturas existentes en la gran mampara de cristal, de $21,64 \times 15,54 \mathrm{~m}$, que se extiende verticalmente, del suelo al techo, constituyendo una delicada división.

Esta monumental mampara, proyectada por el propio Sir Basil Spence y realizada por el artista John Hutton, consta de numerosos paneles, que llevan grabadas figuras de ángeles y santos, y la efigie de San Miguel, en posición central, dominando la 
composición. Fue diseñada pensando en que debía proporcionar la sensación de esbeltez y sutil trasparencia y, además, permitir a los peatones que atraviesen el porche una clara visión del interior, logrando atraer su atención-quizás razonó el Arquitecto: el no religioso que circule a través del porche, entre alguna vez en el interior, que le será de este modo ya familiar-.

A la derecha, entrando en el recinto, se encuentra el baptisterio. Este presenta una gran vidriera-de $24,40 \times 12,20 \mathrm{~m}$-, realizada según un diseño "abstracto" de John Piper, pero de un efecto fuertemente evocador de la vitalidad del nacimiento y del milagro de la Creación. El citado artista, mediante un adecuado aprovechamiento de la energía del color, ha conseguido un rico marco para la pila bautismal.

A la izquierda, y directamente en frente del baptisterio se halla la capilla de la Unidad: adopta en planta una forma de estrella, simbolizando la estrella de Belén, $y$ cuyo perfil es una reminiscencia de las antiguas tiendas de los cruzados, como queriendo recordar que la Unidad es una nueva cruzada. Va cubierta con una bóveda de hormigón, de $10 \mathrm{~cm}$ de espesor, que presenta como elementos sustentantes diez parteluces, asimismo de hormigón, que dejan entre sí unos espacios en los cuales van adosadas unas vidrieras diseñadas por Margaret Traherne. Su pavimento fue ejecutado según el bosquejo del artista sueco Einar Forseth, y ofrece una parte central con la paloma, simbolizando al Espíritu Santo, de la que irradian representaciones emblemáticas de los cuatro Evangelistas, los cinco Continentes y los signos del Zodíaco.

La nave central fue proyectada con la idea de lograr la concentración de la atención de los fieles sobre el altar y el inmenso tapiz-diseñado por Graham Sutherland-existente detrás del mismo. Ayuda a conseguirlo plenamente la sensación de convergencia que producen los muros, debido a su disposición en zigzag, y el sentido direccional dado a la iluminación, mediante la adecuada colocación de los ventana- 
les, ocupando los ángulos entre los muros, que presenta las siguientes ventajas: iluminan eflcientemente la nave, y no molestan al asistente a los actos litúrgicos, ni cuando entra, ni durante la celebración de los mismos.

Lawrence Lee, Keith New y Geoffrey Clarke fueron los diseñadores de las vidrieras de estos ventanales: representan la evolución y el progreso del hombre hacia la perfección que Dios desea para él y a la que el hombre debe siempre aspirar.

Para ayudar a la representación de las distintas fases de la vida del hombre, han sido utilizados acertadamente los recursos que proporciona la "Teoría del Color": así, en la representación de la niñez predominan los verdes frescos; en la pubertad y pasiones de la juventud se utilizan con preferencia los rojos; en la de la madurez, mediante el empleo de una amplia "paleta" se muestra la constante lucha contra el mal, simbolizada por San Miguel y el dragón; para la de la vejez se han reservado los púrpuras oscuros, azules y listas de plata y oro, $y$, finalmente, rodeando al gran altar aparecen los tonos dorados, simbolizando la vida después de la muerte, en la representación del "más allá".

Detrás de la zona del presbiterio, y con accesos independientes por los laterales de la nave central, están la capilla de "La Señora", la de "Cristo de Gethsemani" y la conocida con el nombre de "La Industria"-de planta circular-.

La cripta aloja: la sala capitular-para 30 miembros del Capítulo-, la sacristía, despachos, dependencias auxiliares, etc.

El ediflcio dispone de las más perfectas y modernas instalaciones de luminotecnia y acústicas, previéndose, asimismo, instalaciones especiales para poder emitir por televisión los actos que se celebren en el interior de la catedral. La cublerta es una magniflca realización de la ingeniería moderna en hormigón pretensado, y el intradós es de madera. 
Entre las fachadas exteriores, en situación prominente, aparecen las esculturas de San Miguel y el demonio - la última gran obra religiosa de Sir Jacob Epstein--.

Corona el edificio la "aguja", de diseño abstracto, que se eleva $24 \mathrm{~m}$ sobre la parte superior de la cubierta, como una fiel expresión del espíritu de resurgimiento religioso en Inglaterra.

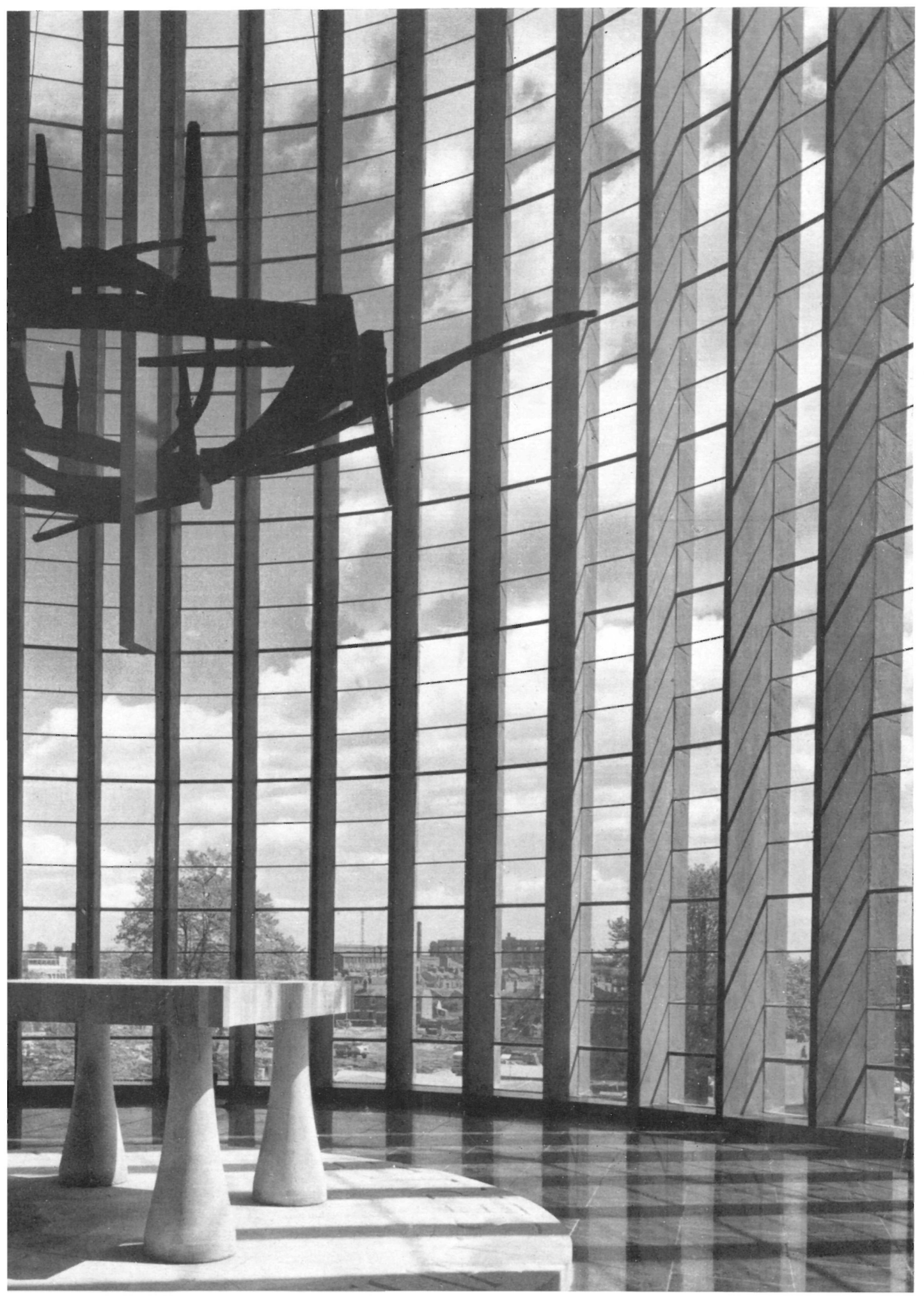


detalles dle intheriores
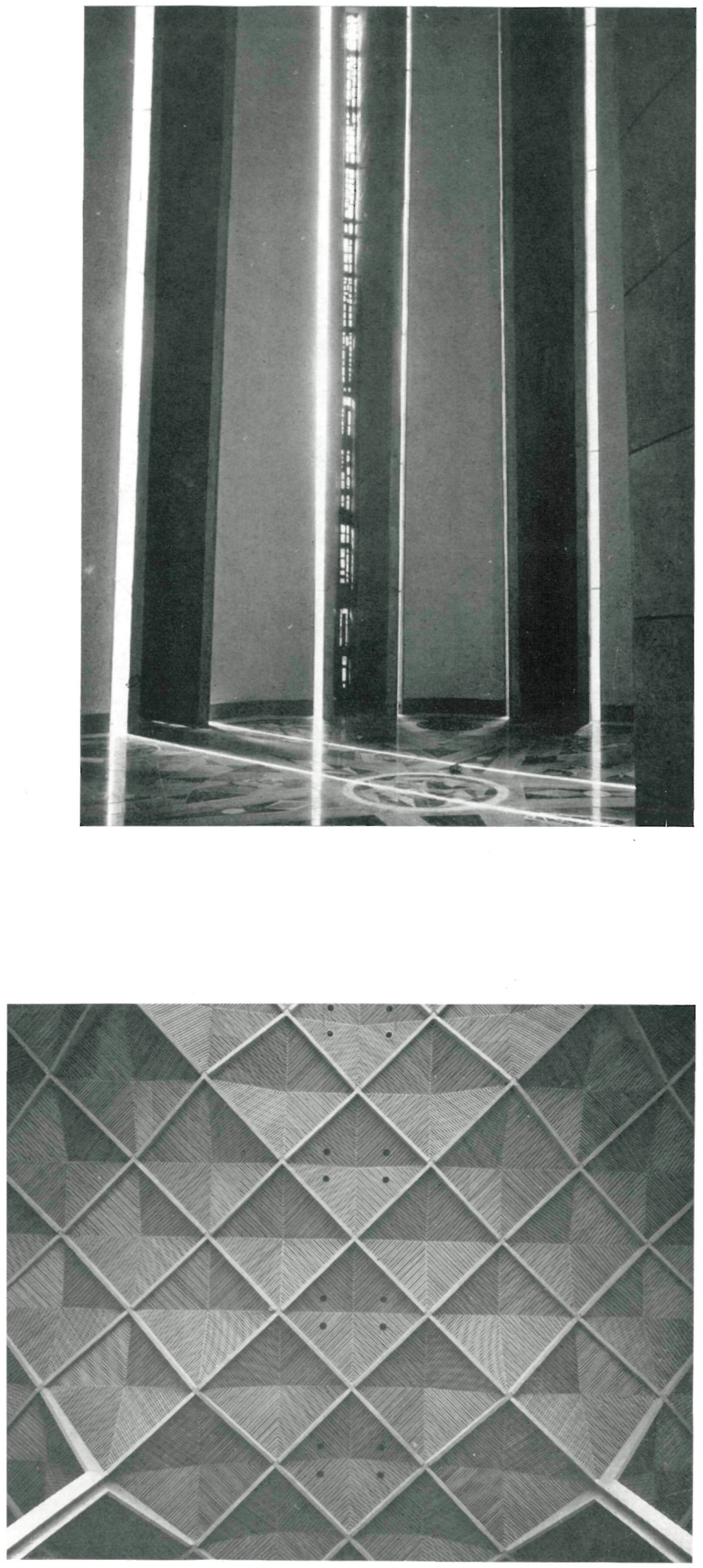

Fotos: HENK SNOEK
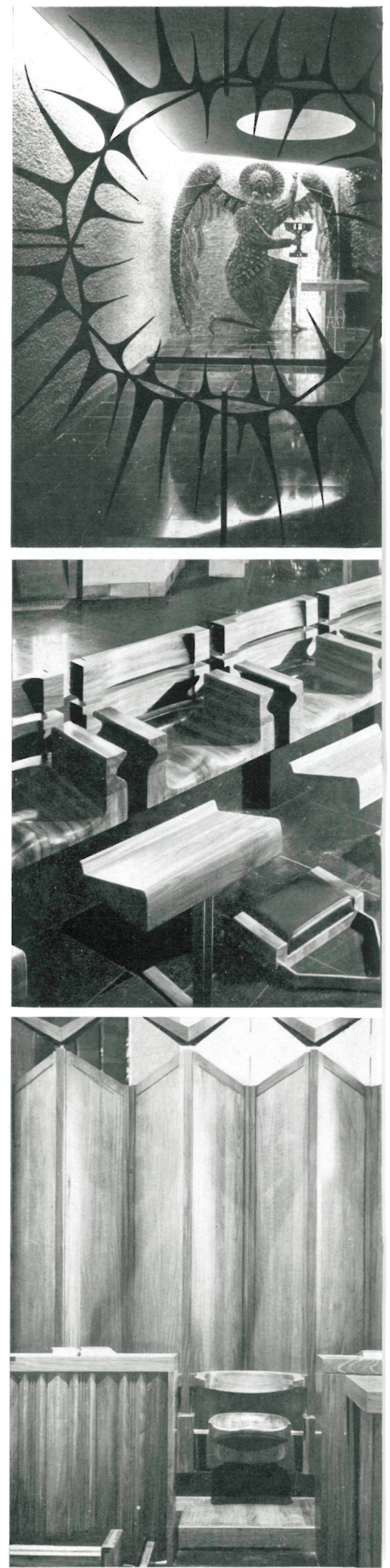


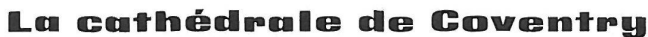

Sir Basil Spence, architecte.

La nouvelle cathédrale de Coventry est un bâtiment de tracé moderne et pondéré, conçu dans Ie respect de I'histoire. EIIe a une capacité de 1.350 fidèles assis et de 2.000 lorsque la nef centrale est totalement pleine, et mesure environ $82,36 \mathrm{~m}$ de long et $24,40 \mathrm{~m}$ de large.

L'entrée principale est un porche aménagé à travers les ouvertures de la grande verrière gravée par John Hotton, qui permet une claire vision de l'intérieur de la nef.

A droite, à l'entrée, se trouve le baptistère-éclairé par un vitrail richement polychromé-et à gauche, la chapelle de l'Unité, en forme d'étoile.

La nef centrale a été conçue dans l'intention de concentrer l'attention des fidèles vers le maître-autel, grâce à la convergence des murs en zigzag et à la direction de l'éclairage.

Derrière le presbytère se trouvent les chapelles connues sous les noms de «La Dame», «Le Christ à Gethsémani» et «L'industrie».

La crypte abrite: la salle capitulaire, la sacristie, bureaux, dépendances auxiliaires, etc.

Entre les façades extérieures, se dresse le groupe de Saint Michel et le démon, oeuvres du sculpteur Sir Jacob Epstein. L'édifice est couronné par «l'aiguille», de conception abstraite, qui s'élève de $24 \mathrm{~m}$ sur la partie supérieure de la couverture, comme la fidèle expression du renouveau de l'esprit religieux en Angleterre.

\section{Coverntring Cathedrand}

Sir Basil Spence, architect.

The new Coventry Cathedral is a modern design, treated with much respect for historical tradition. It can accom modate 1350 seated people, and can hold a congregation of 2000 when the central nave is filled. Its length is $82.36 \mathrm{~m}$, and its width $24.40 \mathrm{~m}$.

The main entrance is protected by a porch, and is an opening in the great glazed curtain wall, engraved by John Hutton, which provides a clear view of the inside of the building.

On entering, to the right, is the baptistry which is illuminated by a richly couloured glass window, and on the left is the Chapel of Unity, whose planform is star shaped.

The central nave was thought out with the idea that the congregation should concentrate its attention on the main altar. This has been successfully done by the zigzag progress of the side walls, and the direction of the illumination.

The chapels of Our Lady, Christ at Gethsemane, and The Industry are behind the Presbitery.

In the crypt there is a chapter room, the sacristy, offices and auxiliary facilities.

In prominent positions, on he outside elevations, there are sculptures of Saint Michael and the Devil, by Sir Jacob Epstein.

The building is crowned by a needle, of abstract design, which rises $24 \mathrm{~m}$ above the top of the roof, and provides a good expression of the resurgence of religious spirit in Britain.

\section{Die Marthedrale vom Coventring}

Sir Basil Spence, Architekt.

Die neue Kathedrale von Coventry ist ein in modernen ausgeglichenen Linien entworfenes Gebäude, bei dem man auch die Geschichte berücksichtigt hat. Sie enthält 1350 Sitzplätze und kann bei grosser Fülle bis zu 2000 Gläubige im Hauptschiff aufnehmen. Sie ist ungefähr $82,36 \mathrm{~m}$ lang und $24,40 \mathrm{~m}$ breit.

Vor dem Haupteingang befindet sich eine von John Hutton entworfene gläserne Vorhalle.

Wenn man reinkommt befindet sich gleich rechts das Taufbecken erleuchtet durch ein farbenreiches Glasfenster und links, die Einigkeitskapelle mit einem sternförmigen Grundriss.

Das Haupschiff wurde mit der Absicht entworfen, die Aufmerksamkeit der Gläubigen ausschliesslich auf den Altar zu lenken, was man durch ein Zusammenlaufen der Mauern im Zick-Zack und die ebenfalls zum Altar hingerichtete Beleuchtung erreicht hat.

Hinter dem Hauptaltar befinden sich die Nebenkapellen, die unter dem Namen «Die Jungfrau», "Christus von Gethsemane» und "Christus als Diener» bekannt sind.

Die Crypta enthält den Kapitelsaal, die Sakristei und Büro- und Nebenräume.

Auf den Aussenfassaden befinden sich die Skulpturen vom heiligen Michael und vom Teufel, die von Sir Jacob Epstein geschaffen wurden.

Gekröhnt wird die Kathedrale durch die sogenannte «Nadel» in abstrakter Form, die sich $24 \mathrm{~m}$ über das Dach der Kathedrale hinaus als Wahrzeichen des Widerauflebens des religiösen Geistes in England erhebt. 